\title{
Functional characterization of the phospholipase C activity of Rv3487c and its localization on the cell wall of Mycobacterium tuberculosis
}

\author{
Madduri Srinivas $^{1}$, Sona Rajakumari ${ }^{2}$, Yeddula Narayana ${ }^{1}$, Beenu Joshi $^{3}, \mathrm{~V} \mathrm{M} \mathrm{Katoch}^{3}$, \\ Ram RajaseKharan ${ }^{2}$ and Kithiganahalli N BaLAJI ${ }^{1, *}$ \\ ${ }^{1}$ Department of Microbiology and Cell Biology, \\ ${ }^{2}$ Department of Biochemistry, Indian Institute of Science, Bangalore 560 012, India \\ ${ }^{3}$ National Jalma Institute of Leprosy and other Mycobacterial Diseases, Tajganj, Agra 282 001, India \\ *Corresponding author (Fax, +91-80-23602697; Email, balaji@mcbl.iisc.ernet.in)
}

\begin{abstract}
Mycobacterium tuberculosis survives and persists for prolonged periods within its host in an asymptomatic, latent state and can reactivate years later if the host's immune system weakens. The dormant bacilli synthesize and accumulate triacylglycerol, reputed to be an energy source during latency. Among the phospholipases, phospholipase $\mathrm{C}$ plays an important role in the pathogenesis. Mutations in a known phospholipase C, plcC, of M. tuberculosis attenuate its growth during the late phase of infection in mice. Hydrolysis of phospholipids by phospholipase C generates diacylglycerol, a well-known signalling molecule that participates in the activation of extracellular signal-regulated kinases (ERK) through protein kinase $\mathrm{C}$ leading to macrophage activation. In the present study, we show that M. tuberculosis possesses an additional cell wall-associated protein, Rv3487c, with phospholipase C activity. The recombinant Rv3487c hydrolyses the substrate phosphatidylcholine and generates diacylglycerol by removing the phosphocholine. Furthermore, Rv3487c is expressed during infection as it exhibits significant humoral immunoreactivity with sera from children with tuberculosis, but not with that from adult patients.
\end{abstract}

[Srinivas M, Rajakumari S, Narayana Y, Joshi B, Katoch V M, Rajasekharan R and Balaji K N 2008 Functional characterization of the phospholipase C activity of Rv3487c and its localization on the cell wall of Mycobacterium tuberculosis; J. Biosci. 33 221-230]

\section{Introduction}

Mycobacterium tuberculosis, the causative agent of pulmonary tuberculosis (TB), infects one-third of the world's population (Murray et al 1990). Despite the multiplicity of antimicrobial mechanisms mounted by its host, $M$. tuberculosis shows a remarkable ability to survive either by evoking survival strategies or by interfering with critical macrophage functions that are required to successfully respond to infection (Gehring et al 2004; Wang et al 2005; Pai et al 2003). M. tuberculosis has a unique ability to persist within the host in a latent state and undergo reactivation years later if the host's immune system weakens
(Deretic and Fratti 1999; Manabe and Bishai 2000). In fact, at this stage, the pathogen is difficult to target. Many studies have suggested that pathogenic mycobacteria primarily subsist on fatty acid substrates during infection and that fatty acids may be the primary source of energy during latency (Daniel et al 2004). In this respect, the bacilli's lipases can play an important role in the release and utilization of host fatty acids. Among the various lipases, phospholipases play an important role as virulence factors in a variety of bacterial infections. They are generally classified into phospholipases A1, A2, C and D based on the cleavage position of the glycerol backbone in the phospholipids (Songer 1997). Among these groups, phospholipase $\mathrm{C}$ plays a significant

Keywords. Cell wall; Mycobacterium tuberculosis; phospholipase C

Abbreviations used: BSA, bovine serum albumin; DAG, diacylglycerol; ERK, extracellular signal-regulated kinases; HRP, horseradish peroxidase; IPTG, isopropyl $\beta$-D-1-thiogalactopyranoside; PBS, phosphate buffered saline; PCR, polymerase chain reaction; TB, tuberculosis; TLC, thin-layer chromatography 
part in the pathogenesis of a variety of bacteria (McNamara et al 1994; Songer 1997) as it generates a signalling lipid molecule $s n$-1,2-diacylglycerol (DAG), a protein kinase C activator in macrophages and other cell types. In addition, DAG is an activator of sphingomyelinase which leads to increased amounts of an important effector molecule, ceramide, which in turn activates MEK (mitogen-activated protein kinase/extra cellular signal-regulated kinase kinase) through protein kinase $\mathrm{C}$ leading to subsequent extracellular signal-regulated kinases (ERK) activation (Monick et al 1999; Cuschieri et al 2006). Activation of the abovementioned mitogen-activated protein kinases represents an important signalling event that regulates the secretion of a variety of cytokines and chemokines by macrophages. In this regard, it has been reported that the growth of a triple plcABC mutant of $M$. tuberculosis was attenuated during the late phase of infection in mice (Raynaud et al 2002).

Earlier studies have reported that the promoter for Rv3487c was upregulated at a low $\mathrm{pH}$ and M. tuberculosis strains deficient in Rv3487c exhibited an attenuated phenotype in the lung of mice in comparison with virulent strains (Saviola et al 2003; Camacho et al 1999). Recently, it was reported that $\mathrm{Rv} 3487 \mathrm{c}$ (LipF) is a lipase with carboxyesterase activity (Zhang et al 2005). In this study, we demonstrate that Rv3487c possesses additional phospholipase $\mathrm{C}$ activity and localizes to the cell wall of $M$. tuberculosis. During infection due to tubercle bacilli, Rv3487c elicits clinical stage-specific humoral immunoreactivity with sera from children with tuberculosis, but not with sera from adult patients.

\section{Materials and methods}

\subsection{Cells and reagents}

Cloning vector pGEMT Easy was purchased from Promega, USA. DNA-modifying enzymes, including restriction endonucleases, polymerases and ligase were procured from Fermentas, USA. Ni-NTA His binding resin, protein purification column and gel extraction kit were from Qiagen. Middlebrook 7H9 was obtained from Difco, USA. Cell wall, cytosol, whole cell lysate and culture filtrate protein fractions of virulent $M$. tuberculosis were obtained from TB research materials and vaccine testing contract, Colorado State University, USA. Most chemicals were obtained from Sigma Aldrich except when otherwise specified. $\left(9,10-{ }^{3} \mathrm{H}(\mathrm{N})\right)$ triolein $(10 \mathrm{Ci} / \mathrm{mmol})$, [dipalmitoyl-1$\left.{ }^{14} \mathrm{C}\right]$ phosphatidylcholine $(54 \mathrm{mCi} / \mathrm{mmol})$ and [2-palmitoyl9,10- $\left.{ }^{3} \mathrm{H}\right]$ phosphatidylcholine $\quad(92.3 \quad \mathrm{Ci} / \mathrm{mmol})$ were obtained from Perkin Elmer Biosystems. Phospholipase C from Bacillus cereus was obtained from Sigma Chemical Company, USA. Thin-layer chromatography (TLC) plates (Silica Gel 60 F-254) were from Merck, USA.

\subsection{Bacterial strains and culture conditions}

For cloning and expression experiments, E. coli JM109, DH5 $\alpha$, and BL21(DE3)/pLysS (Novagen) were used as host strains and cultured in Luria Bertani broth or agar, as required, at $37^{\circ} \mathrm{C}$, supplemented with $100 \mu \mathrm{g} \mathrm{ml}{ }^{-1}$ ampicillin. Mycobacterium smegmatis strain $\mathrm{mc}^{2} 155$ was grown in Middlebrook $7 \mathrm{H} 9$ at $37^{\circ} \mathrm{C}$, supplemented with $0.05 \%$ Tween $80(\mathrm{v} / \mathrm{v}), 0.2 \%$ glycerol $(\mathrm{v} / \mathrm{v})$.

\subsection{Synthesis of phospholipids}

$\left.{ }^{32} \mathrm{P}\right]$ Phospholipids were biosynthesized from mung bean (Vigna radiate) by in vivo labelling with $\left[{ }^{32} \mathrm{P}\right]$ orthophosphate (Herman and Chrispeel 1980). Briefly, $5 \mathrm{~g}$ of mung been seeds were soaked in distilled water for $2-3 \mathrm{~h}$ and then germinated on damp wet cotton in the presence of $20 \mu \mathrm{Ci}$ of $\left.{ }^{32} \mathrm{P}\right]$ orthophosphate for 2 days. The incorporation of $\left[{ }^{32} \mathrm{P}\right]$ orthophosphate was stopped by washing the seeds in cold water followed by boiling the seeds in $10 \%$ acetic acid in isopropyl alcohol. The seeds were then ground in a mortar and pestle and lipids were extracted according to the described protocol (Gangar et al 2002). A fraction of the extracted lipids in chloroform was applied on TLC plates and separated using chloroform:methanol:acetone:acetic acid:water $(50: 10: 20: 15: 5, \mathrm{v} / \mathrm{v})$. The region of the TLC plate corresponding to standard spots, phosphatidylcholine, phosphatidylethanolamine and phosphatidic acids were scraped independently and lipids were extracted from the silica. The incorporation of radioactivity was measured using a scintillation counter. Phospholipids were quantified by digesting the organic phosphate with perchloric acid and the digested phosphate was determined colorimetrically.

\subsection{Thin-layer chromatography}

Phospholipids were chromatographed twice by silica-TLC in the same direction with petroleum ether $\left(30-60^{\circ} \mathrm{C}\right)$ :anhydrous acetone $(1: 3, \mathrm{v} / \mathrm{v})$ followed by chloroform:methanol:ammonia $(65: 35: 2.5, \mathrm{v} / \mathrm{v})$. Neutral lipids were chromatographed with diethyl ether:petroleum ether $\left(30-60^{\circ} \mathrm{C}\right)$ :glacial acetic acid (80:30:1, v/v). Silica-TLC plates (Merck, USA) were used in both chromatography systems. Lipids were routinely located by iodine vapour staining and, on occasion, by contact autoradiography with a Kodak X-ray film. Lipids were identified by comparing them with authentic standards obtained from commercial sources. All substrates were found to be chemically pure by these methods.

\subsection{Cloning and expression of Rv3487c}

The coding sequence of Rv3487c was amplified by polymerase chain reaction $(\mathrm{PCR})$ and cloned into the 
pGEMT Easy vector from the BAC clone containing genomic DNA of $M$. tuberculosis with forward primer containing flag epitope 5'-gcggatccatggactacaaagacgatgacg acaagcttgtlgcgtgcgectggggtgcg-3' and reverse primer 5'-gctc tagagataggcgactgtccaaacg-3'. After DNA sequence analysis, the Rv3487c open reading frame (ORF) was subcloned into the expression vector pRSET-A. The E. coli BL21 strain carrying recombinant plasmids was induced with isopropyl $\beta$ D-1-thiogalactopyranoside (IPTG) at 0.5 OD. Recombinant histidine-flag-tagged Rv3487c protein was expressed and purified under denaturing conditions with an Ni-NTA column (Qiagen, USA). The purified denatured protein was refolded by slow and step-wise dialysis. All lipase assays were performed using the final purified refolded protein. In addition, for the expression of Rv3487c in M. smegmatis, Rv3487c was released from pGEMT-Rv3487c as a BamH1Xbal fragment and subcloned into the expression vector pJAM2, which utilizes the highly inducible acetamidase promoter of M. smegmatis (Triccas et al 1998).

\subsection{Generation of polyclonal antiserum to Rv3487c}

Eight-week-old rabbits were immunized with purified Rv3487c protein with Freund incomplete adjuvant to raise rabbit polyclonal antibodies. The primary subcutaneous immunization was with $1 \mathrm{mg} / \mathrm{ml}$ of purified Rv3487c protein in Freund incomplete adjuvant and, after a period of 30 days, a booster dose of Rv3487c protein $(1 \mathrm{mg} / \mathrm{ml}$ in Freund incomplete adjuvant) was given. After another 15 days, a second booster of Rv3487c $(1 \mathrm{mg} / \mathrm{ml})$ was given prior to the collection of rabbit antiserum.

\subsection{Construction of recombinant $\mathrm{M}$. smegmatis express- ing Rv3487c and subcellular localization of Rv3487c}

In order to express Rv3487c in M. smegmatis, the pJAM2Rv3487c construct was electroporated into M. smegmatis $\mathrm{mc}^{2} 155$ strain and the recombinant Rv3487c protein was induced with $2 \%$ acetamide for $24 \mathrm{~h}$ at $37^{\circ} \mathrm{C}$. The expression level of Rv3487c was confirmed by SDS-PAGE and by immunoblotting with rabbit polyclonal antiserum. In order to detect the subcellular localization of Rv3487c, recombinant M. smegmatis expressing Rv3487c was harvested after acetamide induction and was resuspended in breaking buffer $(2 \% \mathrm{v} / \mathrm{v}$ Triton $\mathrm{X}-100$ in phosphate buffered saline [PBS]) at $0.5 \mathrm{gl}^{-1}$ of wet weight. Cells were lysed by ultrasonication $(10 \times$ of a 60 s cycle with 2 min cooling period between each burst). Unbroken cells were removed by centrifuging at 6000 $g$ for $5 \mathrm{~min}$ and the cell lysate was centrifuged at $27000 \mathrm{~g}$ at $4^{\circ} \mathrm{C}$ for $30 \mathrm{~min}$. In addition, the supernatant was subjected to an ultracentrifugation step at $100000 \mathrm{~g}$ to separate the contaminating membranes and the supernatant obtained after this step was used as the cytosolic fraction. To further remove cytosolic contaminants, the pellet was incubated overnight in breaking buffer. Finally, the washed insoluble cell wall material was obtained by centrifuging at $27000 \mathrm{~g}$ at $4^{\circ} \mathrm{C}$. Silver staining and western blot analysis were performed to identify the cellular localization of this protein by using these cellular fractions. To detect the presence of Rv3487c protein, $5 \mu \mathrm{g}$ of different cellular fractions were further subjected to western blot analysis. We utilized a control antibody, a rabbit polyclonal antibody reactive to another mycobacterial antigen, in our studies and the results clearly demonstrated the absence of cross-reactivity with Rv3487c (data not shown).

\subsection{Substrate preparation}

For triolein hydrolysis, a measured amount of $\left[{ }^{3} \mathrm{H}\right]$ triolein (500 $000 \mathrm{dpm} /$ assay) in chloroform was dried under a stream of nitrogen and then resuspended in $10 \mathrm{mM}$ Tris$\mathrm{HCl}(\mathrm{pH} 7.5)$ buffer containing $1 \%$ gum Arabic (w/v). The emulsion was sonicated and converted into unilamellar membranes by extrusion through $0.1 \mu \mathrm{m}$ polycarbonate filters. For the mixed micelle preparation, dried lipids, i. e. $\left[{ }^{14} \mathrm{C}\right]$ phosphatidylcholine (220 000 dpm/assay), $\left[{ }^{3} \mathrm{H}\right]$ phosphatidylcholine (250 000 dpm/assay $)$ and $\left[{ }^{32} \mathrm{P}\right]$ phospholipids (110 $000 \mathrm{cpm} /$ assay) were resuspended in the assay buffer containing $10 \mathrm{mM} \mathrm{CHAPS}$. The mixture was sonicated until a clear solution was obtained.

\subsection{Lipase assay}

Release of fatty acids and the corresponding products (monoacylglycerol and DAG) were monitored using $\left[{ }^{3} \mathrm{H}\right]$ triolein. Assay was done in a total volume of $100 \mu 1$ in $10 \mathrm{mM}$ Tris-HCl, $\mathrm{pH}$ 7.5. The mixture was incubated at $30^{\circ} \mathrm{C}$ for $20 \mathrm{~min}$ and stopped by the addition of $50 \mu 1$ of $6 \mathrm{~N}$ $\mathrm{HCl}$ before the addition of $400 \mu \mathrm{l}$ of $\mathrm{CHCl}_{3}: \mathrm{CH}_{3} \mathrm{OH}$ (1: $2, \mathrm{v} / \mathrm{v}$ ). The organic chloroform phase was dried (speed vacuum dryer), lipids were resuspended in a small volume of chloroform and the lipids resolved on thin-layer silica gel plates using petroleum ether:diethyl ether:acetic acid, (70: $30: 1, v / v)$. After the run, the lipids were visualized on TLC either by autoradiography or with iodine vapour, and spots corresponding to the unreacted substrate and products were scraped off and quantified with a liquid scintillation counter. Scintillation fluid consisted of $0.5 \%$ toluene-2,5-diphenyloxazole (PPO) and 0.05\% 1,4-bis[2-(5-phenyloxazolyl)] benzene (POPOP) in toluene $(\mathrm{w} / \mathrm{v})$. Control incubations were carried out for zero time or in the absence of enzyme. The control value was subtracted from the actual assay value and the specific activity (nmol of product formed $/ \mathrm{min} / \mathrm{mg}$ protein) was calculated after the correction. 


\subsection{Phospholipase C assay}

Enzyme activity was assayed by measuring the formation of DAG from phophatidylcholine in the presence of $\mathrm{CaCl}_{2}$. The reaction mixture contained $50 \mathrm{mM}$ Tris- $\mathrm{HCl}, \mathrm{pH} 7.5,5$ $\mathrm{mM} \mathrm{CaCl}, 1 \mathrm{mM}$ PC (220 000-250 $000 \mathrm{dpm} /$ assay), $1 \mathrm{mM}$ CHAPS and the enzyme source $(5 \mu \mathrm{g})$, in a total volume of $100 \mu 1$. The reaction was initiated by the addition of enzyme and it was terminated after $20 \mathrm{~min}$. Lipids were separated by a silica-TLC plate using petroleum ether:diethyl ether:acetic acid $(70: 30: 1, \mathrm{v} / \mathrm{v})$ as the solvent system. The radioactivity in DAG was identified using a Phosphor-Imager (Amersham Biosciences, Molecular Dynamics). The spots of DAGs (1,2-, and 1,3-DAGs) were scraped off from the silica-TLC for the determination of radioactivity by liquid scintillation counting. Phospholipase C from Bacillus cereus was used as a positive control in time course experiments. Control incubations were carried out for zero time or in the absence of enzyme. The control values were subtracted from the actual assay value and the enzyme activity was calculated after correction. Specific activity was calculated using $220-250 \mathrm{dpm} / \mathrm{nmol}$ of DAG formed and it was expressed as $\mathrm{nmol}$ of DAG formed $/ \mathrm{min} / \mathrm{mg}$ protein.

\subsection{Study population}

The study population $(n=211)$ comprised patients with TB reporting to the National Jalma Institute of Leprosy and other Mycobacterial Diseases in Agra, India. The patient population was categorized into different clinical groups, as per the guidelines of the National TB Control Programme, Central TB division, Government of India: (i) group $1(n=$ 94) patients who were diagnosed to have pulmonary TB for the first time and had no history of taking chemotherapy; (ii) group $2(n=30)$ patients who had relapsed; (iii) group $3(n=31)$ patients who had extrapulmonary TB infection. Pulmonary TB was confirmed in patients belonging to groups 1 and 2 by the presence of acid-fast bacilli in at least two initial sputum smear examinations and growth of tubercle bacilli in BACTEC cultures. Patients with active TB were also examined for radiological abnormalities by chest X-ray. Patients in group 3 primarily had abdominal TB and TB meningitis. The diagnosis of extrapulmonary TB was carried out by histological examination as well as culture positivity of the bacillus in specimens obtained from extrapulmonary sites. Samples were obtained from all patients at entry into the study and those from HIV-positive subjects were excluded. The healthy controls included in the study were recruited after radiological and clinical examination to rule out the presence of active TB. Those included in the study gave written consent and the study was carried out after approval from the Institutional Bioethics Committee.

\subsection{Serological characterization of Rv3847}

Enzyme-linked immunosorbent assay (ELISA) was carried out in 96-well microtitre plates (Nunc, USA) with the above-mentioned recombinant $\mathrm{PE}$ proteins. The plates were incubated overnight at $4^{\circ} \mathrm{C}$ with recombinant Rv3487c

(a)

(b)

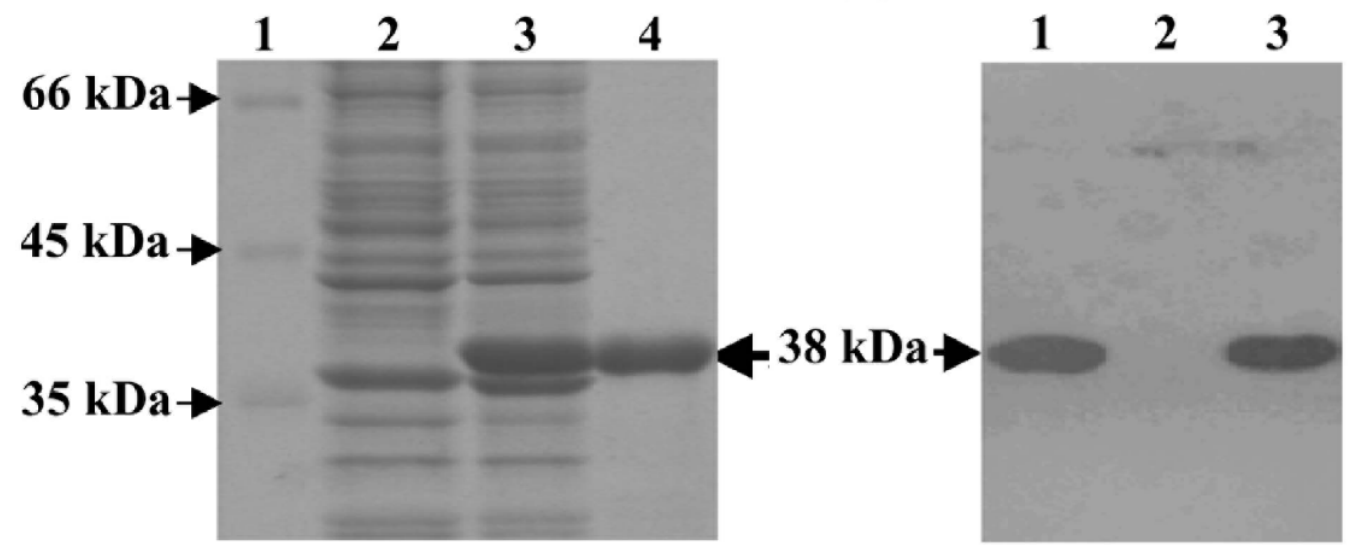

Figure 1. Cloning, expression, purification and generation of polyclonal antibodies of Rv3487c. (a) The Rv3487c gene was PCR amplified with gene-specific primers from an M. tuberculosis BAC clone and cloned into E. coli expression vector pRSET A. Recombinant histidine-flag-tagged Rv3487c protein was expressed and purified by a nickel-nitrilotriacetic acid-agarose column. Lane 1, protein marker; lane 2, uninduced; lane 3, induced; lane 4, purified Rv3487c protein. (b) Immunoblot analysis of the samples described in (a) with Rv3487c rabbit polyclonal antiserum. Lane 1, induced; lane 2, uninduced; lane 3, purified Rv3487c protein. 
protein $(1.25 \mu \mathrm{g} / \mathrm{ml})$ followed by three washes with PBSTween $20(0.05 \%)$ buffer. After blocking with 3\% bovine serum albumin (BSA) in PBS, wells were incubated with human sera (1:400 dilution in blocking buffer) for $1 \mathrm{~h}$ at $37^{\circ} \mathrm{C}$ followed by washing with PBS-Tween 20 buffer. The above-mentioned sera dilution was selected after carrying out careful titration experiments with different dilutions of the patients' sera. The plates were further incubated with anti-human IgG or anti-human IgM-horseradish peroxidase (HRP) followed by developing with o-phenylenediamine tetrahydrochloride. The absorbance values were measured at $492 \mathrm{~nm}$ in an ELISA reader (Molecular Devices, USA).

\subsection{Statistical analysis}

Student $t$ test was used for the analysis of statistical significance ( $P$ value). The data on serological reactivity of different categories of patients were compared with the data from healthy controls. Graphpad Quickcalcs (online $t$ test calculator [http://www.graphpad.com/quickcalcs/ ttest1.cfm]) was used for this purpose. Only $P$ values $<0.05$ were considered significant.

\section{Results}

\subsection{Cloning, expression and purification of Rv3487c}

The recombinant $\mathrm{Rv} 3487 \mathrm{c}$ was cloned, expressed and purified as described in Materials and methods. Figure 1a shows the expression of histidine - flag-tagged Rv3487c protein (lane 3) and purification using nickel-nitrilotriacetic acid agarose (Qiagen) chromatography (lane 4). The purified Rv3487c protein was used to immunize eightweek-old rabbits to generate polyclonal antibodies. Figure $1 \mathrm{~b}$ demonstrates the specific recognition of Rv3487c by polyclonal antibodies in lysates of IPTG-induced, but not in -uninduced, Escherichia coli BL21(DE3)/pLysS cultures carrying pRSET A-Rv3487c (lanes 1-2).

\subsection{Rv3487c exhibits phospholipase C-like activity}

Rv3487c protein has already been shown to have carboxy esterase activity and annotated to be a probable lipase $\mathrm{F}$ (Zhang et al 2005). On the other hand, the same protein was shown to possess no lipase activity (Deb et al 2006). Initial experiments were carried out with radiolabelled triolein to assess the catalytic property of the protein, and it was found that the purified recombinant enzyme had a very weak long chain with triacylglycerol acyl hydrolase activity. We then looked for the hydrolysis of various phospholipids and found that there was a marked reduction of ${ }^{32} \mathrm{P}$-labelled phospholipids (figure 2). These data suggested that Rv3487c protein possessed phospholipase activity and weak or no triacylglycerol acyl hydrolase activity. To find out the type of phospholipase, we assessed the recombinant protein for the hydrolysis of $\left[{ }^{14} \mathrm{C}\right]$ phosphatidylcholine and analysed the hydrolysed products on silica-TLC. As seen in the Phosphor Imager (figure 3a), there was a time-dependent formation of DAGs (1,2-, and 1,3-DAGs) and a corresponding decrease in phosphatidylcholine (origin). These data suggested that the purified recombinant Rv3487c protein hydrolysed the phosphatidylcholine into phosphocholine and DAG. When $\left[{ }^{3} \mathrm{H}\right]$ phosphatidylcholine was incubated with the recombinant Rv3487c enzyme there was a time- (figure $3 b$ ) and protein amount-dependent phospholipase $\mathrm{C}$ type of activity (figure $3 c)$. Commercially available phospholipase $\mathrm{C}$ was used as a positive control using $\left[{ }^{3} \mathrm{H}\right]$ phosphatidylcholine as the substrate (data not shown). However, we are aware that

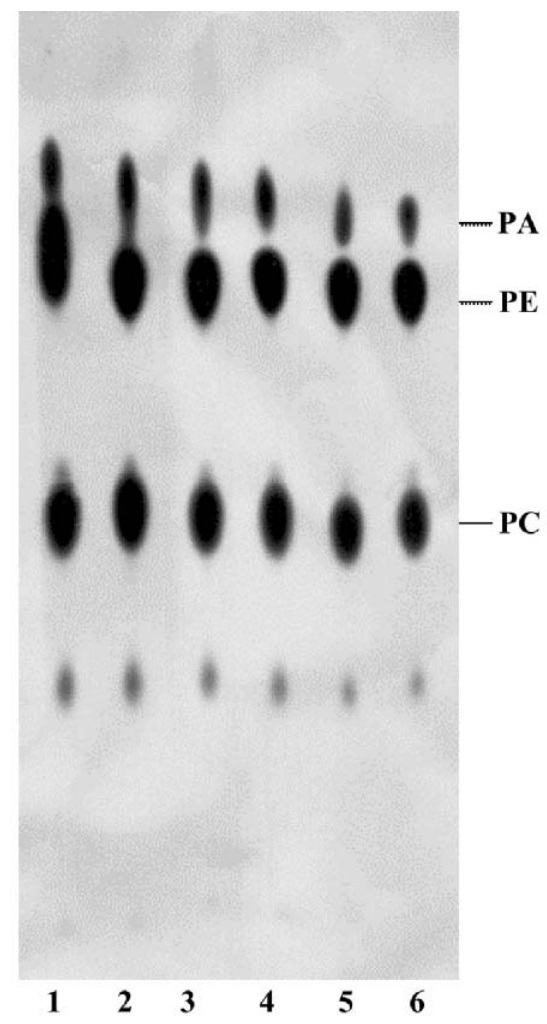

Figure 2. Hydrolysis of $\left[{ }^{32} \mathrm{P}\right]$ phospholipids by the purified recombinant Rv3487c. Phospholipid hydrolysis was conducted by incubating $2 \mathrm{mM}\left[{ }^{32} \mathrm{P}\right]$ phospholipid in the presence of $5.0 \mu \mathrm{g}$ of recombinant Rv3487c protein at different time points, followed by extraction of lipid. Lipid was resolved on silica-thin-layer chromatography (TLC) using chloroform:methanol:acetone:acetic acid:water (170:25:25:25:4, v/v) as the solvent system followed by autoradiography. The TLC experiments were performed twice and a typical autoradiograph is shown. Lane 1 represents no enzyme control; lanes 2-6 correspond to 5, 10, 15, 20 and 30 min reaction time, respectively. PC, phosphatidylcholine; PE, phosphatidylethanolamine; PA, phosphatidic acid. 
(a)

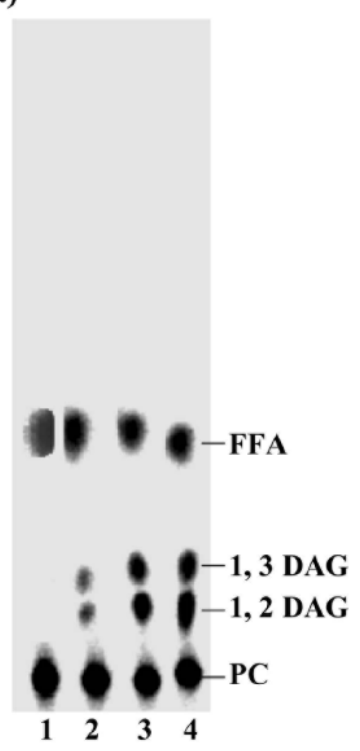

(b)

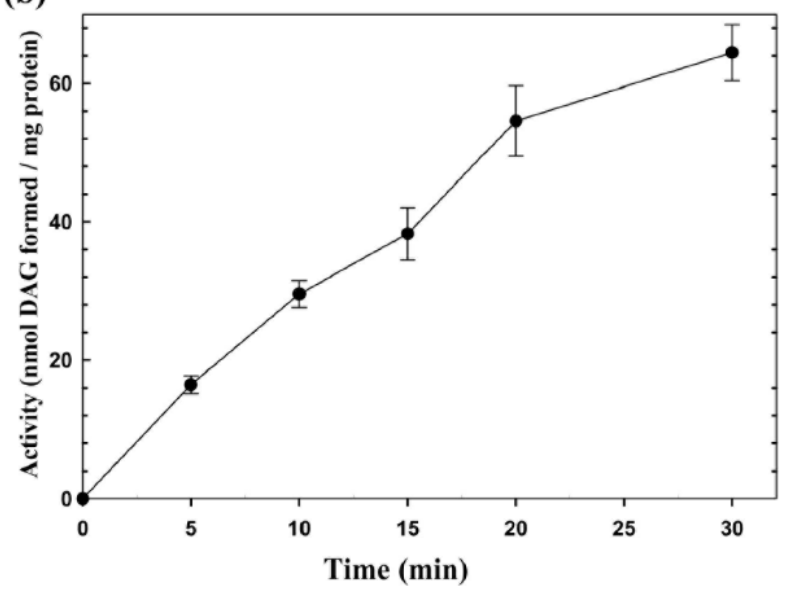

(c)

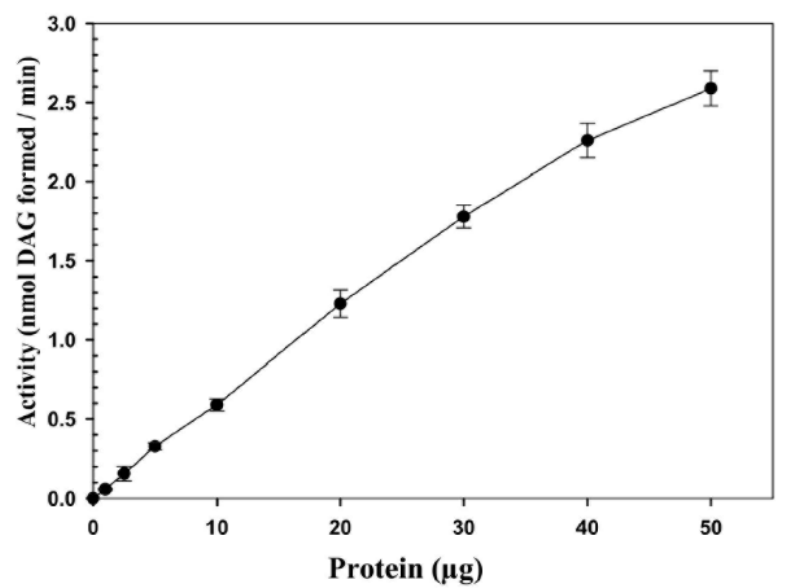

Figure 3. Formation of diacylglycerol (DAG) from phosphatidylcholine by Rv3487c. (a) Phosphatidylcholine hydrolysis was conducted by incubating $1 \mathrm{mM}\left[{ }^{14} \mathrm{C}\right]$ phosphatidylcholine $(220 \mathrm{dpm} / \mathrm{nmol})$ in the presence of $5.0 \mu \mathrm{g}$ of the purified recombinant Rv3487c protein at different time points, followed by lipid extraction. Lipid was resolved on silica-thin-layer chromatography (TLC) using a petroleum ether: diethyl ether:acetic acid (70:30:1, v/v) solvent system followed by imaging by Phosphor imaging system. The TLC experiments were performed three times and a typical PhosphorImage is shown. Lane 1 represents no enzyme control; lanes 2-4 correspond to 5, 10 and 20 min reaction time, respectively. FFA, free fatty acid; 1,2-DAG, 1,2-diacylglycerol; 1,3-DAG, 1,3-diacylglycerol; PC, phosphatidylcholine. (b) Formation of diacylglycerol from $\left[{ }^{14} \mathrm{C}\right] \mathrm{PC}$ was carried out with $5.0 \mu \mathrm{g}$ protein at various time points. (c) Effect of increasing amounts of protein was seen at $30^{\circ} \mathrm{C}$ for 20 min. Values are mean $\pm \mathrm{SD}$ of four independent experiments, each performed in duplicate.

comparing the phospholipase C activity of Rv3487c with a heterologous source to understand the relative activity may not be appropriate. Further, acid-treated (chemical treatment) or heat-killed enzyme was used as a control, which did not demonstrate phospholipase $\mathrm{C}$ activity (data not shown). Taken together, these findings indicate that Rv3487c protein has phospholipase $\mathrm{C}$ activity.

\subsection{Subcellular localization of Rv3487c}

Even though most bacterial phospholipases are secretory proteins, M. tuberculosis phospholipase $\mathrm{C}$ ( $p l c A$, plcB,
$\mathrm{plcC}$ ) is shown to be associated with the cell wall fraction of M. tuberculosis (Raynaud et al 2002). In order to elucidate the subcellular localization of the enzyme, Rv3487c was subcloned into the pJAM2 vector and the pJAM2-Rv3487c construct was electroporated into a M. smegmatis mc $^{2} 155$ strain. In this regard, bioinformatics analysis was carried out to explore whether M. smegmatis possesses Rv3487c and the results suggested that $M$. smegmatis does not have any homologous gene/s with a significant identity or similarity in nucleotide sequence level to Rv3487c. The recombinant M. smegmatis expressing Rv3487c was induced with $2 \%$ acetamide for $24 \mathrm{~h}$ at $37^{\circ} \mathrm{C}$ and different cellular fractions 
were isolated as described earlier (Lee et al 1992; Parish and Stroker 1998). Western blot analysis of these cellular fractions was performed to identify the cellular localization of Rv3487c. Different cellular fractions of virulent $M$. tuberculosis (kind gift from TB research materials and vaccine testing contract, Colorado State University, USA) were also subjected to immunoblotting with polyclonal antibody to Rv3487c. Figure 4a demonstrates that Rv3487c protein is detected in whole cell lysates and cell wall fractions (lanes 1 and 2) of $M$. tuberculosis, but not in culture filtrate proteins. Additionally, subcellular fractions isolated from recombinant $M$. smegmatis expressing Rv3487c (figure 4b, lane 4) suggest that it is associated with the cell wall. The absence of reactivity of the anti-Rv3487c antibody to subcellular fractions of the vector-transformed M. smegmatis (figure 4) clearly suggests that a homologue of Rv3487c might be absent in M. smegmatis.

\subsection{Rv3847c elicits differential B cell responses during TB infection}

The above-mentioned results clearly suggest that Rv3487c is localized to the mycobacterial cell wall leading to the possibility that the Rv3487c protein may be accessible to the immune system of infected individuals. In order to ascertain whether LipF protein is expressed in vivo during active infection with $M$. tuberculosis and that Rv3487c is immunogenic, we assessed the immunoreactivity of Rv3487c with sera derived from different clinical categories of TB patients. Humoral immune responses of group 1 patients (those reporting symptoms of pulmonary TB for the first time) to recombinant Rv3847c protein and 19
$\mathrm{kDa}$ antigens were evaluated along with those of healthy controls as shown in figure $5 \mathrm{a}-\mathrm{d}$. In the case of children with pulmonary TB in group $1, \operatorname{Rv} 3487 \mathrm{c}(P=0.02$, figure 5a) and $19 \mathrm{kDa}$ antigen $(P=0.0174$, figure $5 \mathrm{c})$ showed statistically significant $\mathrm{IgG}$ (figure 5) but not IgM immunoreactivity (data not shown). However, in the case of adult group 1 patients, neither Rv3487c nor $19 \mathrm{kDa}$ antigen elicited significant $\mathrm{IgG}$ antibody reactivity $(P>0.05)$ (figure $5 \mathrm{a}-\mathrm{c}$ ). Furthermore, sera derived from group 2 adult patients with relapse did not demonstrate statistically significant $\mathrm{IgG}$ or IgM reactivity to Rv3487c (data not shown) and sera from children in group 2 were not available for our study. In our study, neither Rv3487c nor $19 \mathrm{kDa}$ antigen elicited IgM reactivity in sera derived from all the three categories of patients (data not shown). The culture filtrate $19 \mathrm{kDa}$ antigen was used in the current study as a positive control, as it is known to elicit strong humoral as well as cell-mediated host immune responses (Harris et al 1991; Faith et al 1991; Lyaschchenko et al 1998).

In the case of group 3 patients (extrapulmonary TB), children but not adults elicited significant IgG immunoreactivity to $\operatorname{Rv} 3487 \mathrm{c}(P=0.004$, figure $5 \mathrm{~b})$ and $19 \mathrm{kDa}$ antigen $(P=0.008$, figure $5 \mathrm{~d})$. In order to compare the serological sensitivities (IgG) of Rv3487c and $19 \mathrm{kDa}$, the data represented in figure 5 were recalculated as the percentage of individuals showing ELISA absorbance values above mean \pm 2 SEM over values of healthy controls. Among all patients, Rv3487c mounted strong IgG antibody responses in $57 \%$ of children in group 1 and $66 \%$ of group 3 individuals, while $19 \mathrm{kDa}$ antigen elicited a response in $56 \%$ of children in group 1 and $63 \%$ of children in group 3 . The observed specific humoral reactivity of sera from children (a)

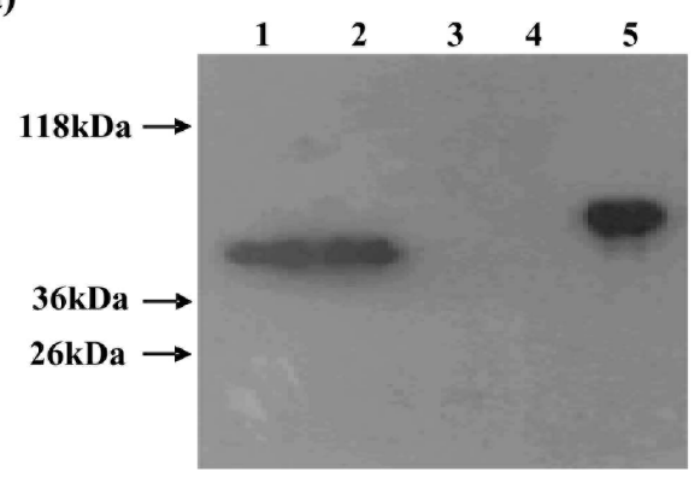

(b)

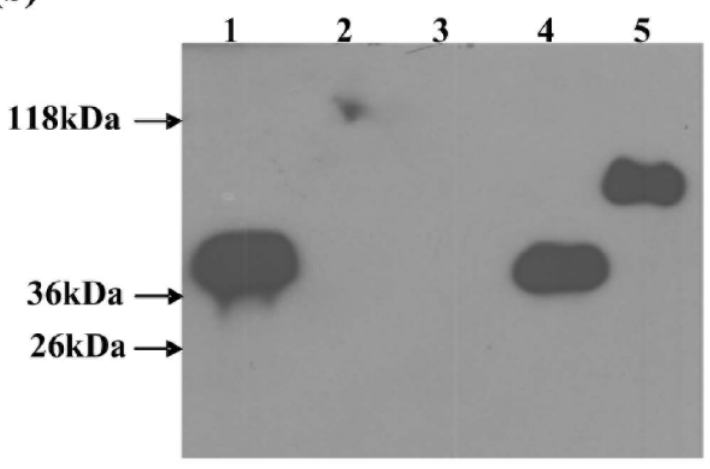

Figure 4. Subcellular localization of M. tuberculosis Rv3487c and construction of recombinant M. smegmatis expressing Rv3487c. Different cellular fractions of virulent M. tuberculosis were probed with anti-Rv3487c polyclonal antibodies to delineate the localization of Rv3487c. (a) Lane 1, whole cell lysate of M. tuberculosis (WCL); lane 2, cell wall of M. tuberculosis (CW); Lane 3, culture filtrate proteins (CFP) of M. tuberculosis; Lane 4, cytosol; Lane 5 represents purified Rv3487c protein. For further confirmation, recombinant $M$. smegmatis expressing Rv3487c was constructed with an acetamide-inducible promoter and different cellular fractions were immunoblotted with antiserum to Rv3487c. (b) Lane 1, whole cell lysate of induced; Lane 2, whole cell lysate of uninduced; Lane 3, cytosol of induced; Lane 4, cell wall of induced M. smegmatis harbouring Rv3487c and Lane 5 represents purified Rv3487c protein. 
(a) $\mathrm{Rv3487c}$

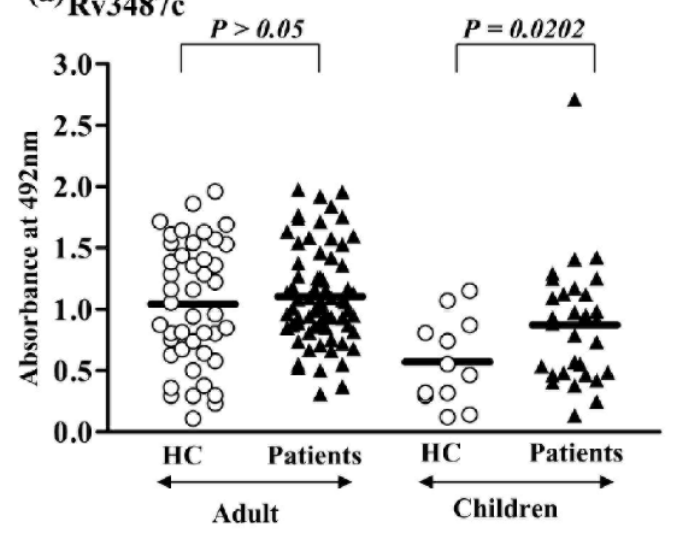

(c) 19-kDa

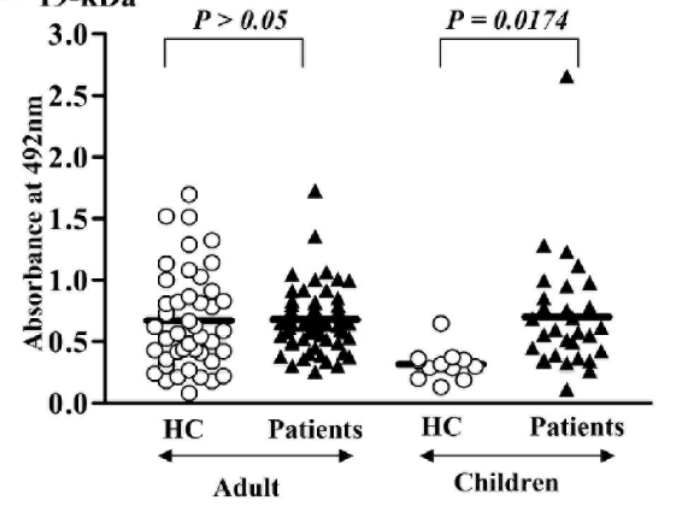

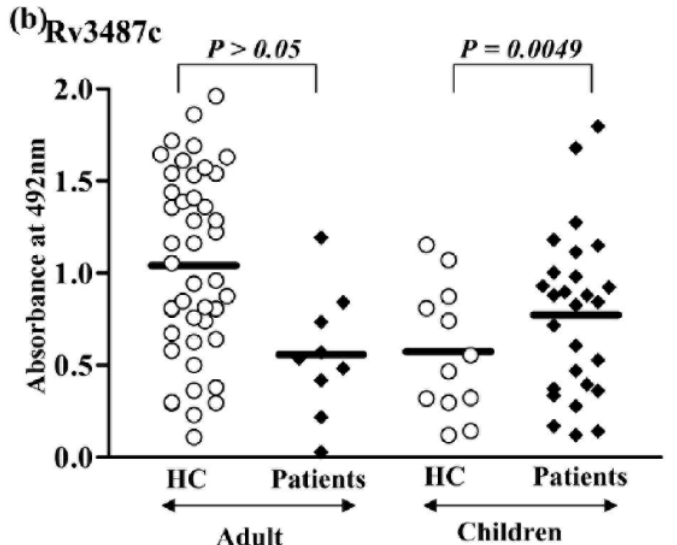

(d)

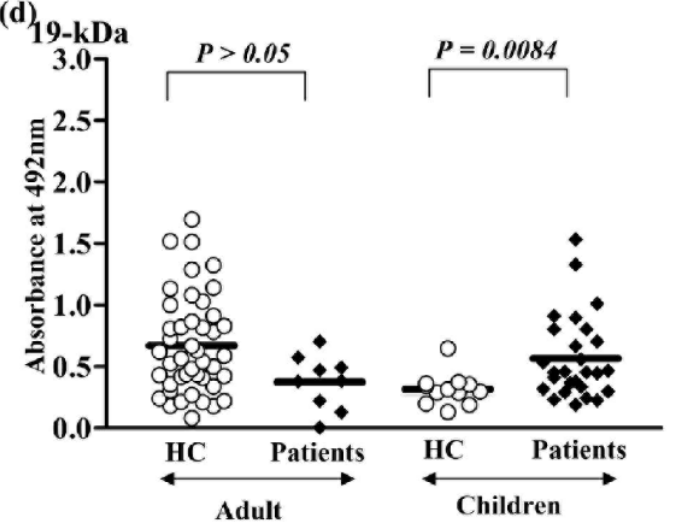

Figure 5. Differential humoral reactivity of Rv3487c antigen with sera derived from different clinical categories of tuberculosis patients. (a-b) Rv3487c elicits a strong and differential antibody response in sera derived from M. tuberculosis-infected children, but not in adult patients or in healthy controls (HC). Rv3487c exhibited strong, statistically significant reactivity to only $\operatorname{IgG}$, but not to $\operatorname{IgM}(P>0.5)$ antibodies in the case of children with tuberculosis reporting symptoms for the first time (group $1, P=0.02$, a) or extrapulmonary infections (group 3, $P=0.004, \mathbf{b})$. (c, d) $19 \mathrm{kDa}$ antigen exhibits immunoreactivity to sera derived from children in groups 1 and 3 , but not to adult patients.

with TB in groups 1 and 3 to Rv3487c, and the relative lack of reactivity to sera derived from adult patients suggest the likelihood of a stage-specific expression of Rv3487c.

\section{Discussion}

Phospholipase genes are induced during infection of human THP-1 derived macrophages with M. tuberculosis. Phospholipase activity is higher in mycobacteria grown in mice than in those grown in lipid-free Dubos medium (Wheeler and Ratledge 1992). The vaccine strain M. bovis BCG and virulent $M$. bovis have only $p l c D$ as the $p l c A B C$ locus is absent and $p l c D$ is suggested to account for the phospholipase $\mathrm{C}$ activity detected in $M$. bovis and $M$. bovis BCG (Gordon et al 1999). Rv3487c is encoded by M. bovis and $M$. bovis BCG. Our results suggest the possibility that Rv3487c, in addition to plcD, can account for the phospholipase $\mathrm{C}$ activity detected in $M$. bovis and $M$. bovis BCG. The enzymatic formation of DAG shown in figure $3 \mathrm{~b}, \mathrm{c}$ clearly represents an important event in the pathogenesis of M. tuberculosis as DAG can act as an important signalling molecule in infected macrophages as well as a precursor for synthesis of triacylglycerol, which acts as an energy store for utilization during long-term dormancy of $M$. tuberculosis (Daniel et al 2004).

It has been reported that Rv3487c (LipF) posses carboxyesterase activity on p-nitrophenyl esters containing a short fatty acyl chain (Zhang et al 2005). However, a recent study reported that Rv3487c (LipF) did not exhibit longchain triacylglycerol (triolein) hydrolysis (Deb et al 2006). Consistent with this later study, we also failed to observe long-chain triacylglycerol hydrolysis (data not shown). Taken together, these results suggest that the esterase activity of Rv3487c is limited to shorter chain substrates. In this respect, Rv3487c exhibits multiple lipase properties 
such as carboxyesterase and phospholipase $\mathrm{C}$ activity. This phospholipase $\mathrm{C}$ activity represents a different class of activity for Rv3487c, which has not been attributed to it earlier.

In addition to phospholipase $\mathrm{C}$ activity, immunoreactivity of Rv3487c with the sera of TB patients as shown in figure 5 clearly suggests its expression during infection. Rv3487c mounted specific and significant humoral immunoreactivity with sera from children in groups 1 and 3, but not with sera derived from adult patients; this suggests the likelihood of stage-specific expression of Rv3487c. These children either had symptoms of TB for the first time (group 1) or had extrapulmonary infection (group 3) with M. tuberculosis. Despite marked elevation of total serum lipids in TB patients, serum phospholipids decrease in concentration in the majority of patients with active pulmonary TB as well as in experimental TB in guinea pigs (Beisel and Fiser 1970). Based on these observations, it possible that Rv3487c expressed during TB infection might utilize serum phospholipids with its phopsholipase $\mathrm{C}$ activity to provide M. tuberculosis with host fatty acids during infection. However, the precise significance of Rv3487c expression in children, but not in adult TB patients, is not very clear. Further, possible reasons for the increased reactivity of Rv3487c in sera from healthy controls between paediatric and adult populations than in the case of patients from the two cohorts are not clear. Clinical stage-specific expression of Rv3487c during infection might have implications for serodiagnosis. Overall, selective expression of Rv3487c might have an important role to play in the virulence of $M$. tuberculosis.

\section{Acknowledgments}

The current study was funded by the Council for Scientific and Industrial Research (CSIR), Department of Biotechnology (DBT), Sir Dorabji Tata Center for Research in Tropical Discases and Department of Science and Technology (DST), New Delhi. Infrastructure support by funding from ICMR (Centre for Advanced Studies in Molecular Medicine), DST (FIST) and UGC (Special assistance) is acknowledged. YN acknowledges the fellowship from CSIR, New Delhi. We would like to thank Professor P Ajit Kumar and Dr $\mathrm{J}$ A Triccas for the pJAM2 vector and Professor Umesh Varshney for his comments during preparation of the current manuscript.

\section{References}

Beisel W R and Fiser R H 1970 Lipid metabolism during infectious illness; Am. J. Clin. Nutr. 23 1069-1079

Camacho L R, Ensergueix D, Perez E, Gicquel B and Guilhot C 1999 Identification of a virulence gene cluster of Mycobacterium tuberculosis by signature tagged transposon mutagenesis; Mol. Microbiol. 34 257-267

Cuschieri J, Billgren J and Maier R V 2006 Phosphatidylcholinespecific phospholipase C (PC-PLC) is required for LPSmediated macrophage activation through CD14; J. Leukoc. Biol. 80 407-414

Daniel J, Deb C, Dubey V S, Sirakova T D, Abomoelak B, Morbidoni H R and Kolattukudy P E 2004 Induction of a novel class of diacylglycerol acyltransferases and triacyl-glycerol accumulation in Mycobacterium tuberculosis as it goes into a dormancy like state in culture; J. Bacteriol. 186 5017-5030

Deb C, Daniel J, Sirakova T D, Abomoelak B, Dubey V S and Kolattukudy P E 2006 A novel lipase belonging to the hormonesensitive lipase family induced under starvation to utilize stored triacylglycerol in Mycobacterium tuberculosis; J. Biol. Chem. $2813866-3875$

Deretic V and Fratti R A 1999 Mycobacterium tuberculosis phagosome; Mol. Microbiol. 31 1603-1609

Faith A, Moreno R, Lathigra R, Roman E, Fernandez M, Brett S, Mitchell D M, Ivanyi J and Rees A D 1991 Analysis of human T cell epitopes in the 19,000 MW antigen of Mycobacterium tuberculosis: influence of HLA-DR; Immunology 74 1-7

Gangar A, Raychaudhuri S and Rajasekharan R 2002 Alteration in the cytosolic triacylglycerol biosynthetic machinery leads to decreased cell growth and triacylglycerol synthesis in oleaginous yeast; Biochem. J. 365 577-589

Gehring A J, Dobos K M, Belisle J T, Harding C V and Boom W H 2004 Mycobacterium tuberculosis LprG (Rv1411c): a novel TLR-2 ligand that inhibits human macrophage class II MHC antigen processing; J. Immunol. 173 2660-2668

Gordon S V, Brosch R, Billault A, Garnier T, Eiglmeier K and Cole S T 1999 Identification of variable regions in the genomes of tubercle bacilli using bacterial artificial chromosome arrays; Mol. Microbiol. 32 643-655

Harris D, Vordermeier H, Roman E, Lathigra R, Brett S J, Moreno C and Ivanyi J 1991 Murine T cell stimulatory peptides from the 19-kDa antigen of Mycobacterium tuberculosis: epitope restricted homology with the $28 \mathrm{kDa}$ protein of Mycobacterium leprae; J. Immunol. 147 2706-2712

Herman E M and Chrispeel M J 1980 Characteristics and subcellular localization of phospholipase $\mathrm{D}$ and phosphatidic acid phosphatase in mung bean cotyledons; Plant Physiol. 66 1001-1007

Lee B Y, Hefta S A and Brennan B J 1992 Characterization of the major membrane protein of virulent Mycobacterium tuberculosis; Infect. Immun. 60 2066-2074

Lyashchenko K, Colangeli R, Houde M, Al Jahdali H, Menzies D and Gennaro M L 1998 Heterogeneous antibody responses in tuberculosis; Infect. Immun. 66 3936-3940

Manabe Y C and W R Bishai 2000 Latent Mycobacterium tuberculosis persistence, patience, and winning by waiting; Nat. Med. 6 1327-1329

McNamara P J, Bradley G A and Songer J G 1994 Targeted mutagenesis of the phospholipase D gene results in decreased virulence of Corynebacterium pseudotuberculosis; Mol. Microbiol. 12 921-930

Monick M M, Carter A B, Gudmundsson G, Mallampalli R, Powers L S, Unninghake G W 1999 A phosphatidylcholinespecific phospholipase $C$ regulates activation of $\mathrm{p} 42 / 44$ 
mitogen-activated protein kinases in lipopolysaccharide-stimulated human alveolar macrophages; J. Immunol. 162 3005-3012

Murray C J L, Styblo K and Rouillon A 1990 Tuberculosis in developing countries: burden, intervention and cost; Bull. Int. Union. Tuberc. Lung Dis. 65 95-96

Pai R K, Convery M, Hamilton T A, Boom W H and Harding C V 2003 Inhibition of IFN- $\gamma$-induced class II transactivator expression by a $19-\mathrm{kDa}$ lipoprotein from Mycobacterium tuberculosis: a potential mechanism for immune evasion; J. Immunol. 171 175-184

Parish T and Stroker N G (eds) 1998 Mycobacteria protocols; in Methods in molecular biology (Totowa, New Jersey, Besrags: Humana Press) pp 91-108

Raynaud C, Guilhot C, Rauzier J, Bordat Y, Pelicic V, Manganelli R, Smith I, Gicquel B and Jackson M 2002 Phospholipases C are involved in the virulence of Mycobacterium tuberculosis; Mol. Microbiol. 45 203-217

Saviola B, Woolwine S C and Bishai W R 2003 Isolation of acidinducible genes of Mycobacterium tuberculosis with the use of recombinase based in vivo expression technology; Infect. Immun. 71 1379-1388

Songer J G 1997 Bacterial phospholipases and their role in virulence; Trends Microbiol. 5 156-161

Triccas J A, Parish T, Britton W J and Gicquel B 1998 An inducible expression system permitting the efficient purification of a recombinant antigen from Mycobacterium smegmatis; FEMS Microbiol. Lett. 167 151-156

Wang Y, Curry H M, Zwilling B S and Lafuse W P 2005 Mycobacteria inhibition of IFN- $\gamma$ induced HLA-DR gene expression by upregulating histone deacetylation at the promoter region in human THP-1 monocytic cells; J. Immunol. 174 5687-5694

Wheeler P R and Ratledge C 1992 Control and location of acylhydrolysing phospholipase activity in pathogenic mycobacteria; J. Gen. Microbiol. 138 825-830

Zhang M, Wang J D, Li Z F, Xie J, Yang Y P, Zhong Y and Wang H H 2005 Expression and characterization of the carboxyl esterase Rv3487c from Mycobacterium tuberculosis; Protein Expr. Purif. 42 59-66

MS received 20 September 2007; accepted 29 January 2008

ePublication: 28 March 2008

Corresponding editor: SEYEd E HASNAIN 